Editorial

\title{
Role of protein ingestion on regulating skeletal muscle protein synthesis
}

\begin{abstract}
Abbreviations: MPS, muscle protein synthesis; IRS, insulin receptor substrate; Akt, protein kinase $\mathrm{B}$; mTORC1, complex 1 of the mechanistic target of rapamycine; P70S6K, phosphorylated protein s6 kinase; eIF4, eukaryotic initiation factors-4; mRNA, messenger RNA
\end{abstract}

\section{Editorial}

The ability of protein ingestion to increase skeletal muscle protein synthesis (MPS) appears to be attributed, in part, to an increase in amino acids available for uptake into skeletal muscle, thereby augmenting substrate availability for MPS. Protein ingestion can up-regulate MPS based on two mechanisms of action. The first is when various amino acids, liberated upon protein digestion in the gut and transported into portal circulation, stimulate the pancreas to release insulin. At this point, insulin can bind to its transmembrane receptor in skeletal muscle. This is a receptor tyrosine kinase which then autophosphorylates insulin receptor substrate 1 (IRS-1), and then activates the downstream cell signaling pathway which contains the protein intermediates, protein kinase B (Akt) and, at the membrane of the lysosome, complex 1 of the mechanistic target of rapamycin (mTORC1). At this point, the activation of subsequent down-stream protein intermediates, phosphorylated protein S6 kinase (P70S6K) and the complex of eukaryotic initiation factors-4 (eIF4) can assist in the formation of the initiation complex on the start codon on the target messenger RNA (mRNA). The second mechanism of protein ingestion bypasses the insulin receptor-mediated signaling cascade up-stream of mTORC1, and this is when leucine is transported into muscle by way of its sodium-dependent amino acid transporter. Leucine then interacts with, and activates, leucyl t-RNA synthetase and this enzyme then interacts with a complex of Rag GTPase proteins. At this point, the Rag GTPases are activated and then interact with mTORC1 at the membrane of the lysosome. Upon mTORC1 activation, the down-stream effectors are the same as those with the insulin-mediated activation of mTORC1 in regard to translation initiation and subsequent MPS. Regarding the amount of protein to effectively stimulate MPS, there are scientific studies showing that large amounts of protein ingested are no more effective at increasing MPS than a less amount of protein., ${ }^{1,2}$ These studies demonstrated that, following resistance exercise, protein ingestion of 20 grams was optimal for stimulating MPS, and that ingesting 40 grams of protein was no more effective than 20 grams. Interestingly, both studies found that protein doses greater than 20 grams resulted in an overabundance of available amino acids that subsequently resulted in an increased rate of amino acid oxidation and ureagenesis. This suggest the scenario of the "muscle full effect" and is based on the standpoint that an upper limit of amino acid delivery must be achieved before muscle can no

\author{
Volume 4 Issue I - 2016
}

\author{
Darryn SWilloughby \\ Department of Health, Baylor University, USA
}

Correspondence: Darryn SWilloughby, Editor-in-Chief, Journal of Nutritional Health and Food Engineering and Exercise and Biochemical Nutrition Lab, Baylor University, Waco,TX, USA, Tel 2547103504, Email darryn_willoughby@baylor.edu

Received: January 26, 2016 | Published: January 28, 2016

longer use amino acids as substrate for muscle protein synthesis, and instead resulting in the amino acids being diverted toward catabolic processes instead.

The Journal of Nutritional Health and Food Engineering is devoted to employing a credible peer-review process in order to publish articles that deal with a number of issues relative to both the basic and applied aspects of nutrition, nutritional health, and food science and engineering. The journal strives to become a high-impact journal with significant relevance in the fields of nutritional health and food engineering.

\section{Acknowledgements}

None.

\section{Conflict of interest}

Author declares that there is no conflict of interest.

\section{References}

1. Moore DR, Robinson MJ, Fry JL, et al. Ingested protein dose response of muscle and albumin protein synthesis after resistance exercise in young men. Am J Clin Nutr. 2009;89(1):161-168.

2. Witard OC, Jackman SR, Breen L, et al. Myofibrillar muscle protein synthesis rates subsequent to a meal in response to increasing doses of whey protein at rest and after resistance exercise. Am J Clin Nutr. 2014;99(1):86-95

3. Atherton PJ, Etheridge T, Watt PW, et al. Muscle full effect after oral protein: time-dependent concordance and discordance between human muscle protein synthesis and mTORC1 signaling. Am J Clin Nutr. 2010;92(5):1080-1088. 